\title{
Percepción sobre actividades de enfermería para satisfacción de necesidades familiares en cuidado intensivo adulto*
}

\author{
Perception on nursing activities to satisfy family needs in the adult intensive care \\ Percepção sobre atividades de enfermagem para satisfação das necessidades familiares em terapia intensiva \\ adulto
}

Edinson Fabian Ardila Suárez ${ }^{a}$

Universidad de Antioquia, Colombia

DOI: https://doi.org/10.11144/Javeriana.ie23.paes

edinson.ardila@udea.edu.co

ORCID: https://orcid.org/0000-0003-0655-3178

Recibido: 07 Junio 2021

Diego Alejandro Salazar Blandon

Aceptado: 14 Octubre 2021

Universidad de Antioquia, Colombia

Publicado: 30 Diciembre 2021

ORCID: https://orcid.org/0000-0002-8724-7705

\section{Resumen:}

Introducción: la satisfacción de las necesidades de la familia en una unidad de cuidado intensivo (UCI) hace parte integral del cuidado del paciente críticamente enfermo y trae beneficios al paciente, la familia y la institución. Objetivo: describir la percepción sobre la frecuencia, pertinencia y viabilidad de actividades de cuidado de enfermería para la satisfacción de las necesidades familiares en UCI. Método: estudio cuantitativo, descriptivo, transversal, realizado a 75 profesionales de enfermería en UCI en un departamento colombiano, mediante muestreo no probabilístico en 11 instituciones hospitalarias de nivel III y IV; la información se recolectó en un periodo de 3 meses, y se aplicó un cuestionario ad hoc diseñado a partir de una revisión integrativa, el cual pasó pruebas por expertos y una prueba piloto; el análisis estadístico se realizó mediante el software SPSS 25.0 y los gráficos mediante el software R, adoptándose como punto de referencia en el análisis una percepción igual o superior al $75 \%$. Resultados: 75 profesionales de enfermería en UCI perciben que, de las 63 actividades propuestas por el cuestionario, 16 se realizan con mayor frecuencia, 27 son muy pertinentes y 24 muy viables. Conclusiones: según los enfermeros de UCI encuestados, basados en la frecuencia, pertinencia y viabilidad, las actividades de mayor importancia son las de satisfacción de las necesidades de seguridad, seguidas de las de información y proximidad, y finalizando por el apoyo y el confort.

Palabras clave: familia, enfermería, unidades de cuidados intensivos, percepción. (Fuente: DeCS, BIREME).

\section{Abstract:}

Introduction: Satisfying the family needs at the intensive care unit (ICU) is an integral part of the critically-ill patient's care, which resolves in benefits to the patient, the family and the health center. Objective: To describe the perception about the frequency, pertinence and suitability of the nursing care activities to fulfil the family needs at the ICU. Methods: It is a cross-sectional, descriptive, quantitative study applied to 75 professional ICU-nurses in a Colombian province under a non-probabilistic sampling in $11 \mathrm{III}$ and IV-level hospitals. Information was gathered during 3 months and an ad hoc instrument designed according to a comprehensive review was used. This instrument underwent both an expert and pilot test. The statistical analyses was conducted using the SPSS 25.0 software and the graphics using the $\mathrm{R}$ software and $\mathrm{a} \geq 75 \%$ perception was adopted as the benchmark in the analysis. Results: Seventy-five professional ICU-nurses perceive that out of 63 activities suggested by the instrument, 16 are carried out more frequently, 27 are more pertinent and 24 very suitable. Conclusions: Based on frequency, pertinence and suitability, the nurses who answered the instrument reported that the most important activities are those intended to satisfy the safety needs, then those intended to inform and getting close, and finally those related to support and comfort.

Keywords: family, nursing, ICU, perception. (Source: DeCS, BIREME).

\section{Resumo:}

Introdução: a satisfação das necessidades da família em uma unidade de terapia intensiva (UTI) é parte integral do cuidado do paciente crítico, trazendo benefícios ao paciente, à família e à instituição. Objetivo: descrever a percepção sobre a frequência, pertinência e viabilidade de atividades de assistência de enfermagem para a satisfação das necessidades familiares na UTI. Método: estudo quantitativo, descritivo, transversal, realizado com 75 profissionais de enfermagem na UTI em um departamento

Notas de autor

$$
\text { a Autor de correspondencia: edinson.ardila@udea.edu.co }
$$


colombiano, mediante amostragem não probabilística em 11 instituições hospitalares de nível III e IV; a informação foi coletada em um período de três meses, e aplicou-se um inquérito ad hoc desenhado a partir de revisão integrativa, o qual passou testes por expertos e teste piloto; a análise estatística foi feita mediante o software SPSS 25.0 e os gráficos mediante o software R, adotando-se como ponto de referência na análise uma percepção igual ou superior a 75\%. Resultados: 75 profissionais de enfermagem na UTI percebem que, das 63 atividades propostas pelo questionário, 16 são realizadas com mais frequência, 27 são muito relevantes e 24 muito viáveis. Conclusões: de acordo com os enfermeiros da UTI pesquisados, com base na frequência, pertinência e viabilidade, as atividades de maior importância são as de satisfação das necessidades de segurança, seguidas das de informação e proximidade, e finalizando com apoio e conforto.

Palavras-chave: família, enfermagem, unidades de terapia intensiva, percepção. (Fuente: DeCS, BIREME).

\section{Introducción}

La unidad de cuidados intensivos (UCI) es un área que ofrece asistencia de soporte vital al paciente críticamente enfermo (1), donde ingresan las personas con enfermedades del patrón epidemiológico propio de cada país. En Colombia, de acuerdo con el análisis de situación de salud (ASIS) de 2019, la disponibilidad de UCI fue de 1868 unidades por cada 100.000 habitantes, comparada con la cifra de 339 en 2014, lo cual sugiere un incremento sustancial en el uso de este servicio (2).

El ingreso de un integrante de la familia a una UCI supone una separación del resto del grupo, y trae consigo repercusiones tanto para el paciente como para su familia (3): implica afrontar situaciones exigentes y cambios en el funcionamiento del núcleo familiar, pues debe resolverse quién se hace cargo de la persona enferma, cómo se manejan a partir de ese momento las reglas en la familia, y se enfrentan nuevas experiencias, sentimientos y responsabilidades, además de los altos niveles de estrés y riesgos en la salud a los que se expone el cuidador familiar por agotamiento (4).

Según (5), dentro de la complejidad de la atención que se brinda en la UCI es importante contemplar también las necesidades de cuidado de su familia, mientras que, por su parte, en (6) se exponen algunas de esas necesidades investigadas en todo el mundo, siendo descritas desde la disciplina de enfermería temáticas de seguridad, información, proximidad, confort y apoyo en la familia de la persona adulta en UCI. Aunado a ello, algunos países han avanzado más en el desarrollo de investigaciones y en propuestas de modelos de atención de enfermería centrados en el cuidado de la familia (7-9).

De acuerdo con la Resolución 2003 de 2014 (10), en Colombia el talento humano de enfermería en la UCI debe tener competencias para brindar una atención segura y de calidad que integre al paciente y su familia; en este mismo contexto, estudios describen que el cuidado a la familia está orientado a la satisfacción de sus necesidades mediante actividades de enfermería, las cuales permiten resultados positivos a nivel fisiológico y psicológico del paciente y su entorno (11-12). Por lo anterior, esta investigación tuvo como objetivo describir la percepción sobre la frecuencia, pertinencia y viabilidad de la actividades de enfermería para la satisfacción de las necesidades familiares en UCI dentro de un contexto colombiano.

\section{Método}

Se realizó un estudio cuantitativo descriptivo y transversal, entre los meses de abril a junio de 2019. La población de estudio estuvo constituida por profesionales de enfermería que laboraban en UCI en un departamento del nororiente colombiano. Se realizó un muestreo no probabilístico a conveniencia por medio de contacto social extrainstitucional, entregándose en formato físico y digital un consentimiento informado y un cuestionario, el cual respondieron 82 profesionales, de los cuales se eliminaron 7 por criterios de inclusión faltantes, cuestionarios incompletos o incorrectamente diligenciados, cargas laborales de menos de 48 horas semanales y desempeño en cargos administrativos. Se contó entonces con una muestra de 75 profesionales provenientes de 11 de las 12 instituciones de salud de nivel III y IV presentes en el departamento. 
Para la recolección de la información se diseñó un cuestionario ad hoc (debido a que no se encontró, al momento de la investigación, un instrumento que cumpliera con el objetivo de esta) con dos apartados: el primero contenía datos sobre variables sociodemográficas y experticia profesional; el segundo se realizó basándose en una revisión integrativa (13) que encontró 64 actividades de cuidado para la familia de pacientes adultos hospitalizados en UCI en el mundo, organizadas desde la visión de enfermería según las 5 necesidades propuestas por (14) y (15). Se realizaron preguntas intencionadas, para medir desde la percepción de los participantes la frecuencia, pertinencia y viabilidad de cada una de ellas mediante una escala Likert de 4 categorías. El cuestionario resultante fue sometido a una prueba de comprensibilidad y ajuste semántico por 4 expertos (16); estos ajustes sugeridos permitieron eliminar una de las actividades, por no tener sentido en el contexto colombiano. Posteriormente se realizó una prueba piloto a 18 profesionales que cumplieron los criterios (los datos de esta prueba no se incluyeron en la muestra definitiva), con el fin de evaluar el tiempo de diligenciamiento y comprensión semántica, logrando así consolidar las preguntas relacionadas con la frecuencia, pertinencia y viabilidad de las actividades de enfermería dirigidas a las necesidades de seguridad, proximidad, información, confort y apoyo.

Para el análisis se elaboró una base de datos en software estadístico SPSS 25.0, donde las actividades fueron codificadas según las tres primeras letras de la necesidad a la cual pertenecen, seguidas de un número de acuerdo con el orden; se calcularon promedios y desviaciones estándar para las variables cuantitativas, y para las cualitativas se establecieron frecuencias y porcentajes. Para visualizar los resultados de la frecuencia, pertinencia y viabilidad de las 63 actividades se usó un gráfico polar (17), realizado en el software estadístico $\mathrm{R}$; posterior a esto se compararon en una misma tabla las respuestas con un porcentaje superior o igual al 75 $\%$ en las categorías: siempre, muy pertinente y muy viable, con el fin de identificar las actividades específicas realizadas con mayor frecuencia, así como determinar cuáles son pertinentes, pero no tan viables, y cuáles son viables pero no muy frecuentes.

Esta investigación estuvo basada en las pautas éticas propuestas por Ezekiel Emanuel, la clasificación del riesgo de acuerdo con la Resolución 8430 de 1993 y los derechos de autor de la Ley 23 de 1982, requerida para la investigación en humanos, contando además con el aval del Comité de Ética e Investigación de la Facultad de Enfermería de la Universidad de Antioquia, acta CEI-FE 2019-06.

\section{Resultados}

En la tabla 1 se muestra que la edad de la mayoría de los participantes oscila entre 27 y 41 años, predominando el sexo femenino; en la formación académica el pregrado tiene un mayor porcentaje, y los profesionales se desempeñan sobre todo en UCI general, con experiencia de entre 1 y 11 años, principalmente. La mayoría de ellos manifiestan que tienen contacto con la familia del paciente entre 2 y 10 horas semanales, aproximadamente. 
TABLA 1

Variables sociodemográficas, de experiencia y experticia en una muestra de profesionales de enfermería que laboran en UCI en un departamento colombiano, 2019.

\begin{tabular}{|l|l|r|r|r|r|}
\hline \multicolumn{2}{|c|}{ Variables } & Media & D.E & n & $\%$ \\
\hline Edad & Masculino & 34 & 7 & & \\
\hline \multirow{2}{*}{ Sexo } & Femenino & & & 19 & 25,3 \\
\hline \multirow{2}{*}{$\begin{array}{l}\text { Formación } \\
\text { académica }\end{array}$} & Pregrado & & & 56 & 74,7 \\
\cline { 2 - 6 } & Especialización & & 42 & 56,0 \\
\cline { 2 - 6 } & Maestría & & 31 & 41,3 \\
\hline \multirow{2}{*}{ Tipo de UCI: } & General & & 2 & 2,7 \\
\cline { 2 - 6 } & Cardiovascular & 9 & 65 & 86,7 \\
\hline Años de experiencia laboral & 6,1 & 5,4 & & \\
\hline $\begin{array}{l}\text { Años de experiencia laboral como } \\
\text { profesional de enfermería en UCI }\end{array}$ & & & & \\
\hline $\begin{array}{l}\text { Horas al día que tiene contacto con la familia } \\
\text { del paciente aproximadamente (horas) }\end{array}$ & 6 & 4 & & \\
\hline
\end{tabular}

Fuente: Elaboración propia.

En la figura 1 resulta relevante cómo el color morado, referente a la categoría siempre, se hace predominante en la mayoría de las actividades, solo superado por el color rojo (nunca se hace) en 3 actividades de seguridad, relacionadas con la participación familiar en rondas de la UCI (SEG01), reanimación cardiopulmonar o procedimientos invasivos (SEG02) y en la entrega de turno (SEG03). Sin embargo, de las 63 actividades reportadas en la literatura, el $75 \%$ o más de los profesionales manifiestan que siempre se hacen 16 actividades distribuidas según las necesidades de seguridad (7), proximidad (3), información (3), apoyo (2) y confort (1), de donde se infiere que las restantes actividades se realizan con menor frecuencia (casi siempre o algunas veces).

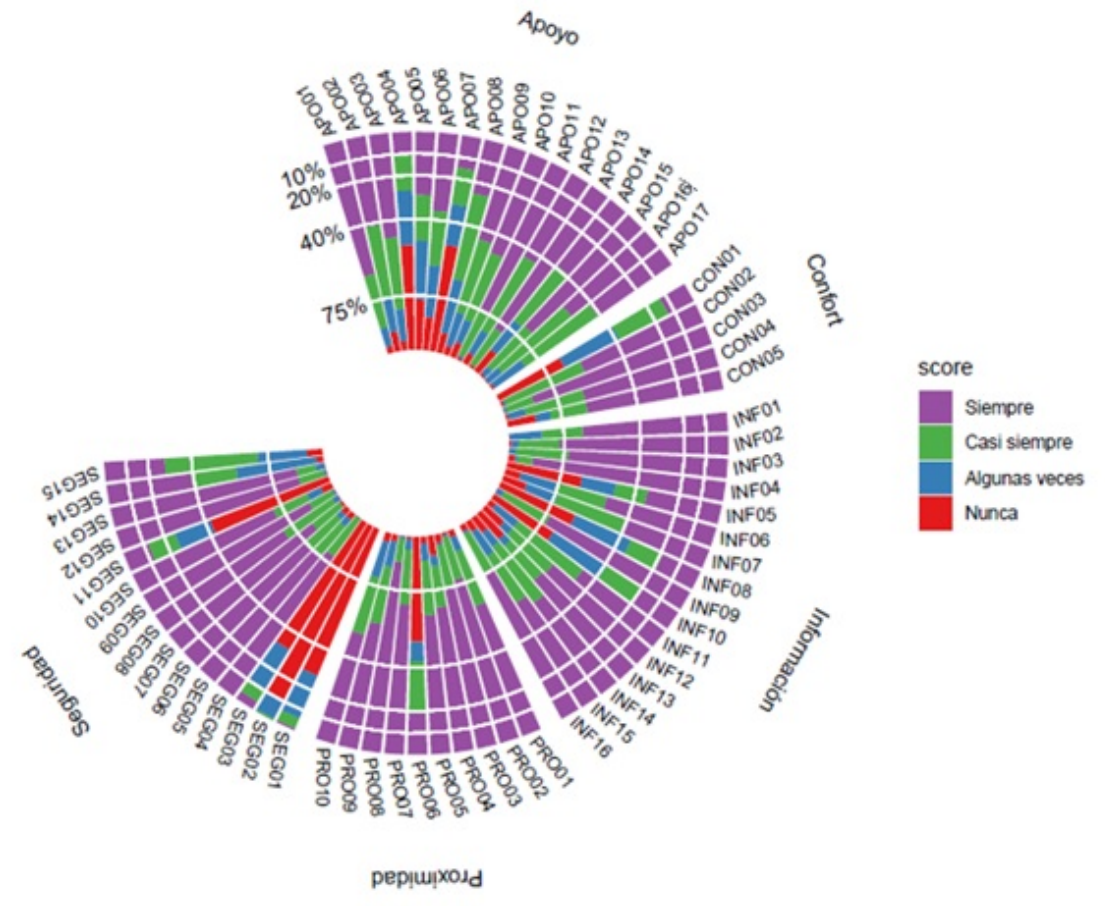

FIGURA 1

Frecuencia de actividades de cuidado a la familia de pacientes adultos hospitalizados en UCI, según la percepción de una muestra de profesionales de enfermería en un departamento colombiano, 2019.

Fuente: Kettunen et al. (17).

Cuando se preguntó a los profesionales sobre la pertinencia de cada una de las actividades para el cuidado de la familia, según su experiencia, la mayoría de estas son evaluadas como pertinentes o muy pertinentes (colores morado y verde en la figura 2). Así, el $75 \%$ o más de los participantes consideran muy pertinentes 9 actividades 
enfocadas a la necesidad de seguridad, 8 de proximidad, 5 de información, 1 de confort y 4 de apoyo, para un total de 27 de las 63 actividades reportadas para el cuidado de la familia de pacientes hospitalizados en UCI. Por su parte, se observa un alto porcentaje de profesionales que valoran el acompañamiento a la familia en la observación de la reanimación cardiopulmonar o de procedimientos invasivos (SEG02) como nada pertinente

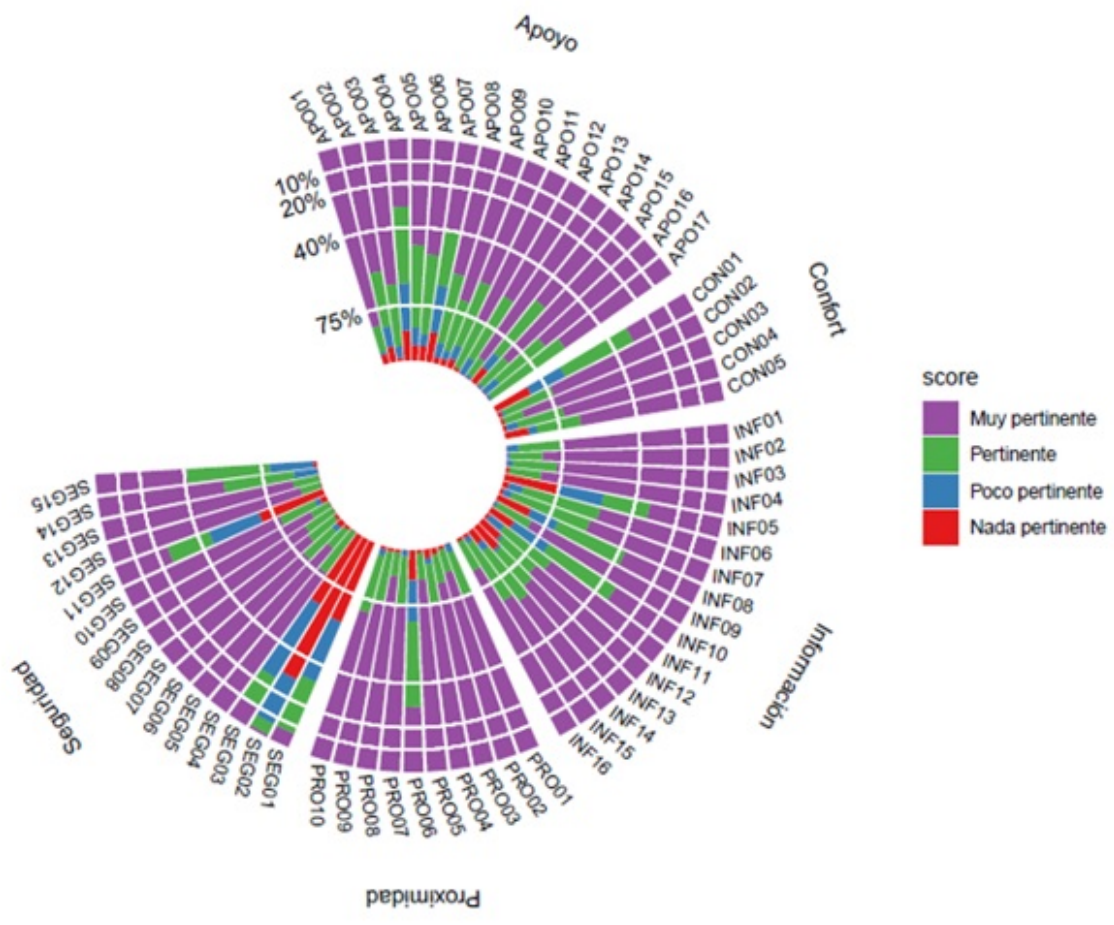

FIGURA 2

Pertinencia de actividades de cuidado a la familia de pacientes adultos hospitalizados en UCI, según la percepción de una muestra de profesionales de enfermería en un departamento colombiano, 2019. Fuente: Kettunen et al. (17).

Frente a la posibilidad de realización de cada una de las actividades según el contexto laboral (viabilidad), la mayoría de las actividades son valoradas como viables o muy viables (figura 3), de modo que el $75 \%$ o más de los participantes concuerdan en calificar como muy viables 7 actividades referentes a la necesidad de seguridad, 6 de proximidad, 5 de información, 2 de confort y 4 de apoyo. Es decir, consideran como muy viables en su entorno de trabajo 24 de las 63 actividades enfocadas al cuidado de la familia; nuevamente, figuran SEG01, SEG02 y SEG03 como nada viables, según la percepción de los enfermeros. 


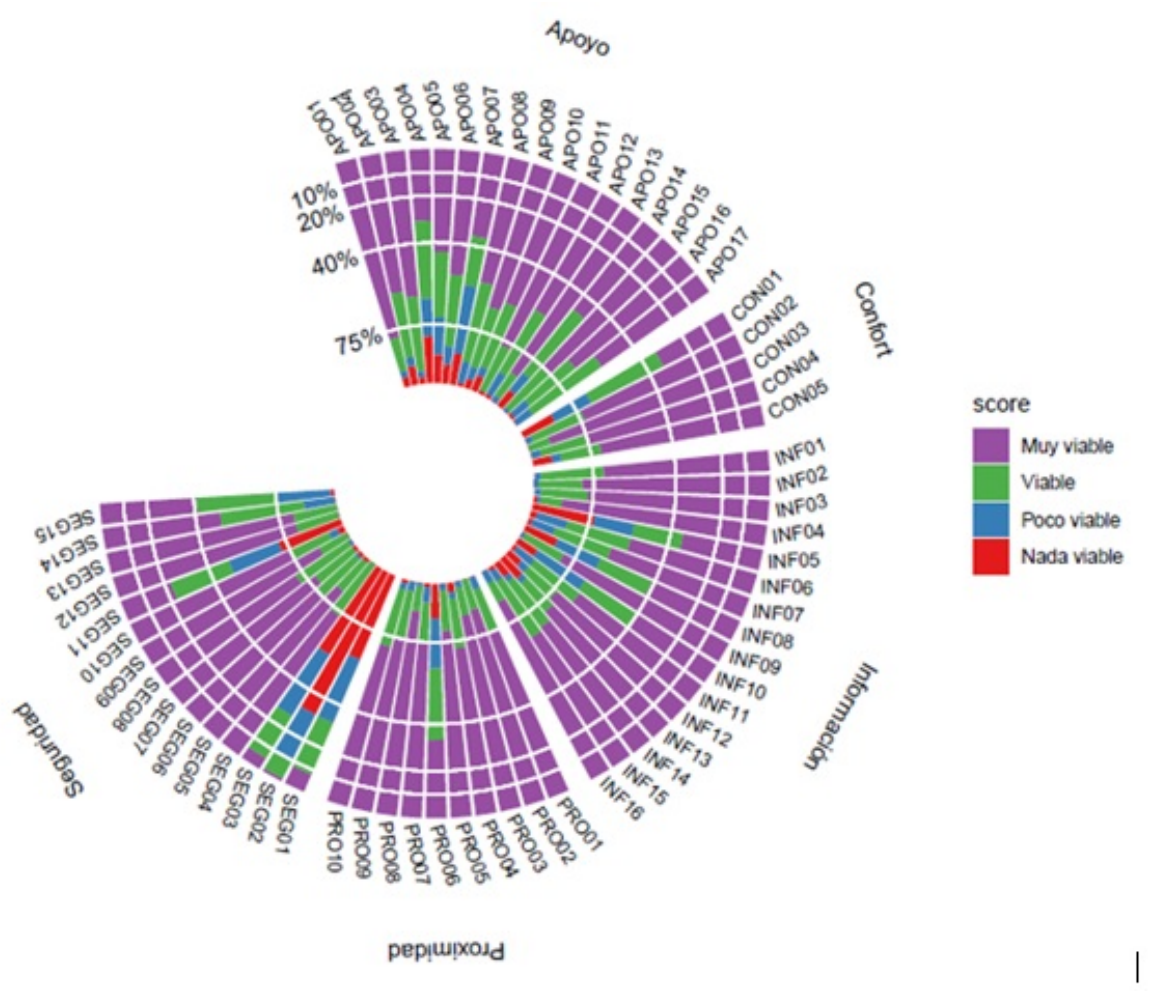

FIGURA 3

Viabilidad de actividades de cuidado a la familia de pacientes adultos hospitalizados en UCI, según la percepción de una muestra de profesionales de enfermería en un departamento colombiano, 2019.

Fuente: Kettunen et al. (17).

En la tabla 2 se establece una comparación entre la frecuencia, pertinencia y viabilidad de las actividades, con una puntuación positiva aproximada de al menos el $75 \%$. De la frecuencia se puede inferir que se realizan en mayor cantidad actividades relacionadas con la dimensión de seguridad, hallándose una percepción similar en la pertinencia y viabilidad; para el personal de enfermería son consideradas como muy pertinentes 27 actividades y como muy viables 24 . Se resaltan dentro de la necesidad de proximidad actividades como permitir la presencia de la familia en los últimos momentos de la vida (PRO02) y su participación el cuidado básico del paciente (PRO05), las cuales, según los resultados, se hacen con una frecuencia menor, en comparación con el porcentaje obtenido en pertinencia y viabilidad; esta misma situación se evidencia en la necesidad de apoyo con la actividad de acompañar a la familia en la muerte del paciente (APO01). 
TABLA 2

Actividades para el cuidado de familias de pacientes adultos hospitalizados en UCI en un departamento colombiano, con puntuación mayor o igual al $75 \%$ en frecuencia, pertinencia o viabilidad.

\begin{tabular}{|c|c|c|c|c|}
\hline Actividad & Código & Siempre & $\begin{array}{c}\text { Muy } \\
\text { pertinente }\end{array}$ & Muy viable \\
\hline \multicolumn{5}{|l|}{ Seguridad } \\
\hline $\begin{array}{l}\text { Mostrar interés por el paciente } \\
\text { y la familia }\end{array}$ & SEG04 & $79 \%$ & $77 \%$ & $73 \%$ \\
\hline $\begin{array}{l}\text { Presentar al personal de } \\
\text { enfermería que labora en la } \\
\text { UCI }\end{array}$ & SEG05 & $76 \%$ & $88 \%$ & $83 \%$ \\
\hline $\begin{array}{l}\text { Asegurar que se mantendrá el } \\
\text { orden de la unidad en ausencia } \\
\text { de los familiares }\end{array}$ & SEG06 & $75 \%$ & $77 \%$ & $76 \%$ \\
\hline $\begin{array}{l}\text { Asegurar que durante la } \\
\text { ausencia de los familiares } \\
\text { todas las necesidades del } \\
\text { paciente se suplirán }\end{array}$ & SEG07 & $83 \%$ & $84 \%$ & $79 \%$ \\
\hline $\begin{array}{l}\text { Hablar diariamente con la } \\
\text { familia del paciente }\end{array}$ & SEG08 & $71 \%$ & $85 \%$ & $72 \%$ \\
\hline $\begin{array}{l}\text { Respetar la confidencialidad } \\
\text { de los miembros de la familia }\end{array}$ & SEG09 & $87 \%$ & $91 \%$ & $87 \%$ \\
\hline $\begin{array}{l}\text { Permitir la participación en la } \\
\text { toma de decisiones de no } \\
\text { reanimación }\end{array}$ & SEG10 & $71 \%$ & $77 \%$ & $75 \%$ \\
\hline $\begin{array}{l}\text { Mantener el contacto visual } \\
\text { con los miembros de la familia } \\
\text { durante el diálogo }\end{array}$ & SEG12 & $87 \%$ & $91 \%$ & $81 \%$ \\
\hline $\begin{array}{l}\text { Asegurar a los familiares que } \\
\text { el personal está bien } \\
\text { capacitado y brinda la mejor } \\
\text { atención }\end{array}$ & SEG13 & $87 \%$ & $88 \%$ & $81 \%$ \\
\hline \multicolumn{5}{|l|}{ Información } \\
\hline $\begin{array}{l}\text { Llamar al miembro principal } \\
\text { de la familia cuando ocurran } \\
\text { cambios en la condición del } \\
\text { paciente }\end{array}$ & INF01 & $67 \%$ & $76 \%$ & $71 \%$ \\
\hline $\begin{array}{l}\text { Dar explicaciones sobre la } \\
\text { enfermedad del paciente } \\
\text { usando términos que sean } \\
\text { comprensibles para la familia }\end{array}$ & INF02 & $77 \%$ & $84 \%$ & $80 \%$ \\
\hline $\begin{array}{l}\text { Responder honestamente las } \\
\text { preguntas que hace la familia }\end{array}$ & INF03 & $73 \%$ & $77 \%$ & $77 \%$ \\
\hline $\begin{array}{l}\text { Entregar información } \\
\text { presencial sobre el paciente al } \\
\text { menos una vez al día }\end{array}$ & INF04 & $89 \%$ & $84 \%$ & $88 \%$ \\
\hline $\begin{array}{l}\text { Aclarar dudas e inquietudes } \\
\text { sobre el estado del paciente y } \\
\text { el entorno }\end{array}$ & INF09 & $73 \%$ & $81 \%$ & $80 \%$ \\
\hline $\begin{array}{l}\text { Incentivar la comunicación } \\
\text { verbal con el paciente para la } \\
\text { orientación en persona, tiempo } \\
\text { y espacio }\end{array}$ & INF16 & $76 \%$ & $84 \%$ & $85 \%$ \\
\hline \multicolumn{5}{|l|}{ Proximidad } \\
\hline $\begin{array}{l}\text { Mantener la presencia de los } \\
\text { familiares cercanos en el } \\
\text { entorno del paciente }\end{array}$ & PRO01 & $64 \%$ & $76 \%$ & $76 \%$ \\
\hline $\begin{array}{l}\text { Permitir la presencia de la } \\
\text { familia en los últimos } \\
\text { momentos de la } \\
\text { vida/despedida del paciente }\end{array}$ & PRO02 & $77 \%$ & $88 \%$ & $87 \%$ \\
\hline $\begin{array}{l}\text { Mantener siempre un dialogo } \\
\text { directo con la familia acerca } \\
\text { de la condición del paciente }\end{array}$ & PRO03 & $75 \%$ & $84 \%$ & $85 \%$ \\
\hline $\begin{array}{l}\text { Permitir a los familiares el uso } \\
\text { de elementos de cuidado } \\
\text { personal como lociones, } \\
\text { cortaúñas y cremas para el } \\
\text { paciente }\end{array}$ & PRO04 & $67 \%$ & $76 \%$ & $72 \%$ \\
\hline $\begin{array}{l}\text { Permitir que la familia ayude } \\
\text { al paciente en algunas cosas } \\
\text { básicas (masajes, higiene } \\
\text { bucal, etc.) del dolor }\end{array}$ & PRO05 & $64 \%$ & $87 \%$ & $80 \%$ \\
\hline $\begin{array}{l}\text { Permitir la proximidad fisica y } \\
\text { las manifestaciones de cariño } \\
\text { entre la familia y el paciente }\end{array}$ & PRO07 & $68 \%$ & $75 \%$ & $75 \%$ \\
\hline $\begin{array}{l}\text { Explicar a la familia que el } \\
\text { paciente puede escucharlos } \\
\text { incluso estando bajo efectos } \\
\text { sedantes o estado de coma }\end{array}$ & PRO08 & $88 \%$ & $88 \%$ & $88 \%$ \\
\hline $\begin{array}{l}\text { Realizar contacto físico } \\
\text { (saludo, realización de } \\
\text { cuidados conjuntos) con los } \\
\text { familiares en la medida de lo } \\
\text { requerido }\end{array}$ & PRO09 & $59 \%$ & $75 \%$ & $76 \%$ \\
\hline \multicolumn{5}{|l|}{ Confort } \\
\hline $\begin{array}{l}\text { Identificar y respetar la cultura } \\
\text { del paciente (religión, cultura, } \\
\text { lenguaje, educación, } \\
\text { comportamiento, expectativas }\end{array}$ & $\mathrm{CON} 03$ & $85 \%$ & $89 \%$ & $91 \%$ \\
\hline
\end{tabular}




\begin{tabular}{|c|c|c|c|c|}
\hline $\begin{array}{l}\text { del rol, ideas sobre salud y } \\
\text { enfermedad, cuidado) }\end{array}$ & & & & \\
\hline $\begin{array}{l}\text { Identificar la fe y las creencias } \\
\text { de los miembros de la familia }\end{array}$ & CON04 & $63 \%$ & $72 \%$ & $76 \%$ \\
\hline \multicolumn{5}{|l|}{ Apoyo } \\
\hline $\begin{array}{l}\text { Brindar apoyo a la familia } \\
\text { frente a la muerte de un ser } \\
\text { querido }\end{array}$ & APO01 & $63 \%$ & $83 \%$ & $79 \%$ \\
\hline $\begin{array}{l}\text { Aceptar las decisiones que } \\
\text { hayan tomado los miembros } \\
\text { de la familia en relación con la } \\
\text { salud y futuro de su paciente }\end{array}$ & APO11 & $75 \%$ & $75 \%$ & $77 \%$ \\
\hline $\begin{array}{l}\text { Escuchar con mucha atención } \\
\text { a los familiares del paciente }\end{array}$ & APO13 & $79 \%$ & $89 \%$ & $85 \%$ \\
\hline $\begin{array}{l}\text { Mostrar empatía por el } \\
\text { paciente y los miembros de la } \\
\text { familia }\end{array}$ & APO15 & $69 \%$ & $83 \%$ & $76 \%$ \\
\hline
\end{tabular}

Fuente: Elaboración propia.

\section{Discusión}

Según la percepción de los enfermeros encuestados, las actividades relacionadas principalmente con la satisfacción de las necesidades de seguridad, información y proximidad son las de mayor frecuencia, pertinencia y viabilidad; esto concuerda con estudios como los realizados en Irán, donde se examinó la percepción de 80 enfermeras sobre las necesidades de las familias, utilizando el inventario de necesidades de Molter (CCFNI), siendo la dimensión de seguridad la de mayor puntaje, e información la segunda (18). También, (19) comparó la percepción de 51 enfermeras sobre las necesidades de la familia en la UCI, concluyendo igualmente que las necesidades más importantes son las de seguridad e información. En Nepal se midió la percepción de 65 enfermeras y de las familias de pacientes admitidos en UCI, identificando del mismo modo que las mayores necesidades de la familia son las de seguridad $(86,25 \%)$ e información $(78,58$ \%) (20). Similarmente, un estudio de Arabia Saudí reporta que las tres principales necesidades de la familia en la UCI son, en orden, seguridad, información y proximidad (21).

En Inglaterra se midió la percepción de las familias y se identificó la comunicación como un aspecto importante en el cual las enfermeras de la UCI tienen un papel central (22), dado que las familias desean información clara y comprensible sobre la condición del paciente, sin dejar espacio a esperanzas poco realistas (23). Así mismo, un estudio adicional concluye que los profesionales de enfermería son facilitadores de la comunicación y de la información, y que las enfermeras son proveedoras de apoyo a la familia, por lo que deben estar dispuestas a ganar su confianza (24). En esta misma dirección se ha demostrado que la satisfacción y la percepción de las necesidades de información son importantes en el contexto de la UCI (25), en sintonía con lo hallado en (26), donde se muestra una correlación positiva entre la información brindada y la satisfacción del cuidado $(r=0,742, p<0,001)$.

Para los profesionales incluidos en el estudio, las actividades que satisfacen las necesidades de seguridad y comunicación relacionadas con la participación de la familia en el cuidado del paciente son viables y pertinentes, lo cual concuerda con (27), donde se refiere que promover la participación familiar en la atención mejora la interacción entre el médico y la familia, y puede reducir el desarrollo de síntomas relacionados con el estrés postraumático después del alta de la UCI (Grado B).

En cuanto a las actividades para brindar soporte a la familia, en (28) se señala que el apoyo hace parte de las competencias profesionales, contribuyendo al cuidado holístico del paciente y la familia. Para ello se deben establecer relaciones de confianza mediante la empatía, pues de este modo se ayuda a la familia y esta a su vez contribuye con el cuidado del paciente. Por su parte, en (29) se diseña un ensayo clínico para comprobar la efectividad de una intervención de apoyo a la familia en una UCI de Estados Unidos: los resultados no mostraron cambios significativos en la ansiedad y depresión hasta 6 meses después. Sin embargo, hubo significancia estadística en la escala de calidad de la comunicación ( $\mathrm{P}=0,001)$, del cuidado centrado en el paciente $(\mathrm{P}=0,006)$ y disminución de la estancia en la $\mathrm{UCI}$ (4,4 días vs. 6,8 días, $\mathrm{P}=<0,001)$. En este sentido, 
según la percepción de los profesionales de enfermería encuestados, las actividades para suplir las necesidades de apoyo son consideras muy pertinentes, a pesar de que se realizan con poca frecuencia.

En cuanto al cuidado por parte de la familia para brindar confort, se encontró que la mayoría de las veces esto se enfoca al bienestar físico relacionado con instalaciones adecuadas para entablar conversaciones o descansar. En (23) se reporta una mayor satisfacción familiar sobre las necesidades de confort después de la implementación de un programa de voluntariado en la UCI, aunque no se encontraron diferencias entre la información, la seguridad, la proximidad y el apoyo. En Villavicencio, Colombia, se establecieron las principales necesidades de la familia en la UCI de acuerdo con el inventario CCFNI medido en 34 familiares (30), concluyendo que en mayor grado de importancia se encuentran las necesidades de seguridad (86\%) e información (82\%), seguidos de proximidad (78\%), apoyo (57\%) y confort (54\%).

Todo esto permite lograr un acercamiento entre las necesidades de la familia y la disposición de los profesionales de enfermería sobre su cuidado. En general, la percepción sobre la frecuencia, pertinencia y viabilidad de las actividades presenta un orden de importancia similar tanto para la familia como para el personal sanitario, siendo este un punto de partida para el diseño y validación de intervenciones que permitan suplir dichas necesidades.

\section{Conclusiones}

Al analizar la frecuencia, pertinencia y viabilidad de las actividades de cuidado de la familia, según la percepción de profesionales de enfermería que laboran en UCI en un departamento del nororiente colombiano, se encuentra que en primer lugar de importancia están las actividades que permiten suplir la seguridad de la familia de adultos críticamente enfermos, seguidas de las actividades para suplir las necesidades de información y proximidad, y luego aquellas para favorecer el confort y el apoyo. Además, son más las actividades que se consideran muy pertinentes y viables que las que se realizan con frecuencia, lo que ofrece un panorama de actividades a considerar en el cuidado de la familia del paciente en UCI para este contexto particular.

\section{Bibliografía}

1. Delgado-Macías JC, Lara-Fajardo VG, Flores-Tacle LC, Sabando-Farías BA, Aguilar-Sánchez EG, FernandezZambrano GA. Patologías específicas de importancia en la U . C . I. Rev Científicade Investig Actual del mundo las ciencias. 2018;3(2):665-87. https://doi.org/10.26820/reciamuc/3.(2).abril.2019.665-687

2. Plata Casas L, Avella Tolosa A, Rodriguez Gutiérrez L. Análisis de situación de salud (ASIS) Colombia, 2019. Ministerio de Salud y Protección Social; 2019. Disponible en: https://www.minsalud.gov.co/sites/rid/Lists/Bi bliotecaDigital/RIDE/VS/ED/PSP/asis-2019-colombia.pdf

3. Márquez-Herrera M, Carrillo-González GM. La experiencia del familiar de la Unidad de Cuidados Intensivos en Bucaramanga (Colombia): un estudio fenomenológico. Rev Arch Med. 2015;15(1):95-106. http://www.reda lyc.org/articulo.oa?id=273840435010

4. Padilla-Fortunatti C, Rojas-Silva N, Amthauer-Rojas M, Molina-Muñoz Y. Necesidades de los familiares de pacientes críticos en un hospital académico de Chile. Enferm Intensiva. 2018;29(1):32-40. https://doi.org/10.1016/j.en fi.2017.09.001

5. Padilla Fortunatti CF. Most important needs of family members of critical patients in light of the Critical Care Family Needs Inventory. Investig Educ en Enferm. 2014;32(2):306-316. http://www.scielo.org.co/scielo.php? script=sci_arttext\&pid=S0120-53072014000200013\&lng=en\&tlng=en.

6. Duque-Delgado L, Rincón E, León-Gómez VE. Apoyo emocional de las familias a los pacientes en Unidades de Cuidados Intensivos. Revisión Bibliográfica.2020;14(3):e14308. http://scielo.isciii.es/scielo.php?script=sci_ar ttext\&pid=S1988-348X2020000300008\&lng=es 
7. Van-Mol M, Boeter GW, Verharen L, Kompanje EJO, Bakker J, Nijkamp MD. Patient- and family-centred care in the intensive care unit: a challenge in the daily practice of healthcare professionals. J Clin Nurs. 2017;26(1920):3212-23. https://doi.org/10.1111/jocn.13669

8. Zaforteza-Lallemand C, Prieto-González S, Canet-Ferrer TP, Díaz-López Y, Molina-Santiago M, Moreno-Mulet $\mathrm{C}$, et al. Mejorando el cuidado a los familiares del paciente crítico: estrategias consensuadas. Enferm Intensiva. 2010;21(1):11-9 https://doi.org/10.1016/j.enfi.2009.07.002

9. Anativia-Montenegro P, Farias-Reyes D, Galiano-Gálvez MA, Quiroga-Toledo N. Visita restrictiva / Visita no restrictiva en una unidad de paciente crítico adulto. Aquichan. 2016;16(3):340-58. http://www.redalyc.org/a rticulo.oa?id=74147078006

10. Díaz-Mass DC, Soto-Lesmes VI. Competencias de enfermeras para gestionar el cuidado directo en la Unidad de Cuidados Intensivos de adultos. Rev Cubana Enfermer. 2020;36(3):e3446. http://scielo.sld.cu/scielo.php?scrip $\mathrm{t}=$ sci_arttext\&pid=S0864-03192020000300019\&lng=es

11. Quijano-Boada LC. Información: La principal necesidad de las familias en Unidad de Cuidados Intensivos (UCI) [Tesis de maestría] Bogotá: Universidad Nacional de Colombia; 2016. https://repositorio.unal.edu.co/handle /unal/58919

12. Motta-Hoyos M, Otalora-Oviedo C, Sierra-Castillo H. Percepción familiar de pacientes de la UCI adulto sobre el cuidado de enfermería en una clínica privada en Florencia Caquetá . Cina Res. 2018;2(1):24-31. https://journ als.uninavarra.edu.co/index.php/cinaresearch/article/view/85

13. Ardila-Suárez EF, Arredondo-Holguín E. Actividades de enfermería para la satisfacción de necesidades familiares en cuidado intensivo adulto: una revisión integrativa. Revista Cuidarte. 2021; 12(1):e1229. http://dx.doi.org/ $10.15649 /$ cuidarte.1229

14. Molter NC. Needs of relatives of critically ill patients\#: a descriptive study. Hear Lung J Acute Crit Care. $1979 ; 8(2): 332-9$.

15. Leske JS. Internal psychometric properties of the Critical Care Family Needs Inventory. Hear Lung J Acute Crit Care. 1991;20(3):236-44.

16. Hernández-Sampieri R, Mendoza-Torres CP. Metodología de la investigación. México DF: McGraw Hill; 2018.

17. Kettunen J et.al. Genome-wide association study identifies multiple loci influencing human serum metabolite levels. Nature Genetics, 2012; 44; 269-276. https://doi.org/10.1038/ng.1073

18. Shorofi SA, Jannati Y, Moghaddam HR, Yazdani-charati J. Psychosocial needs of families of intensive care patients\#: Perceptions of nurses and families. Niger Med J.2016;57(1):10-8. https://doi.org/10.4103/0300-1652.180557

19. Akhlak S, Shdaifat E. Needs of families with a relative in critical care unit. Malaysian J Public Heal Med. 2016;16(3):75-81.

20. Chhetri IK, Thulung B. Perception of nurses on needs of family members of patient admitted to critical care units of teaching hospital, Chitwan Nepal\#: a cross-sectional institutional based study. Nurs Res Pract. 2018; Article ID: 1369164 . https://doi.org/10.1155/2018/1369164

21. Alsharari AF. The needs of family members of patients admitted to the intensive care unit. Patient Prefer Adherence. 2019;13(1):465-73. https://dx.doi.org/10.2147\%2FPPA.S197769

22. Briggs D. Improving communication with families in the intensive care unit. Nurs Stand. 2017;32(2):41-8. http s://doi.org/10.7748/ns.2017.e10812

23. Scott P, Thomson P, Shepherd A. Families of patients in ICU\#: a scoping review of their needs and satisfaction with care. Nurs Open. 2019;6:698-712. https://dx.doi.org/10.1002\%2Fnop2.287

24. Adams AMN, Mannix T, Harrington A. Nurses' communication with families in the intensive care unit - a literature review. Nurs Crit Care. 2017;22(2):70-80. https://doi.org/10.1111/nicc.12141

25. Davidson J. Family-Centered Care meeting the needs of patients' families and hHelping families adapt to critical illnes. Crit Care Nurse. 2009;29(3):28-34. https://doi.org/10.4037/ccn2009611 
26. Bailey JJ, Sabbagh M, Loiselle CG, Boileau J, Mcvey L. Supporting families in the ICU\#: a descriptive correlational study of informational support , anxiety, and satisfaction with care. Intensive Crit Care Nurs. 2010;26(2):11422. https://doi.org/10.1016/j.iccn.2009.12.006

27. Kynoch K, Chang A, Coyer F, McArdel A. The effectiveness of interventions to meet family needs of critically ill patients in an adult intensive care unit: a systematic review update. Joanna Briggs Inst. 2016;14(3)179-232. ht tps://doi.org/10.11124/jbisrir-2016-2477

28. Campo-Martínez M, Cotrina-Gamboa M. Relación de ayuda al familiar del paciente en situación crítica. Enfermería Glob. 2011;24:103-9. https://dx.doi.org/10.4321/S1695-61412011000400009.

29. White D, Angus D, Shields A-M, Buddadhumaruk P, Pidro C, Paner C, et al. A randomized trial of a familysupport intervention in intensive care units. N Engl J Med. 2018;378:2365-75. https://doi.org/10.1056/NEJ Moa1802637

30. Galvis-López CR, Salamanca-Ramos E. Percepción de necesidades en cuidadores familiares de adultos internados en una unidad de cuidados intensivos de una institución prestadora de salud (IPS ) privada en Villavicencio, Colombia. Investig Enferm Imagen Desarr. 2014;16(2):81-94. http://www.redalyc.org/articulo.oa?id=14523 2887006

\section{Notas}

* Artículo original de investigación

\section{Licencia Creative Commons CC BY 4.0}

Limitaciones: Este estudio tiene como principales limitaciones el alcance descriptivo, y el no contar con un instrumento validado para medir el cuidado de enfermería a la familia en la UCI como constructo teórico. Así mismo, el cuestionario diseñado y validado por expertos no cuenta con pruebas psicométricas que permitan reproducirlo; sin embargo, puede ser la base para diseñar y validar instrumentos en el tema.

Financiamiento: La presente investigación no contó con recursos para su realización; el investigador principal contó con financiación para formación posgradual por parte del Departamento de Ciencia, Tecnología e Innovación (Colciencias) y la Gobernación de Santander (Convocatoria 771/2016).

Conflictos de interés: Los autores declaran no tener conflicto de interés.

Cómo citar este artículo: Ardila Suárez E. F., Salazar Blandon D. A. Percepción sobre actividades de enfermería para satisfacción de necesidades familiares en cuidado intensivo adulto. Investigación en Enfermería, Imagen y Desarrollo. 2021;23. https://doi.org/10.11144/Javeriana.ie23.paes 